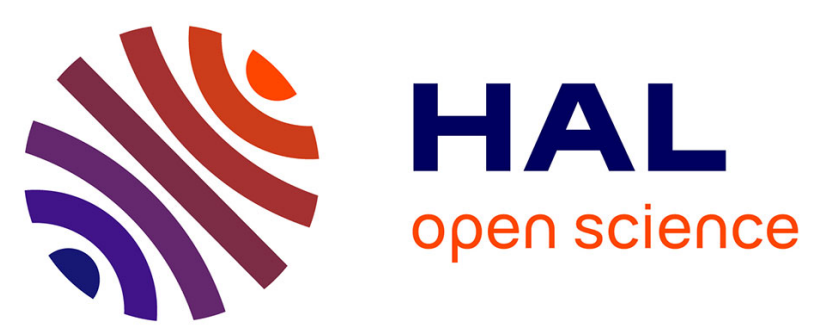

\title{
An Efficient Method to Study the Tradeoff Between Power Amplifier Efficiency and Digital Predistortion Complexity
}

Siqi Wang, Morgan Roger, Julien Sarrazin, Caroline Lelandais-Perrault

\section{- To cite this version:}

Siqi Wang, Morgan Roger, Julien Sarrazin, Caroline Lelandais-Perrault. An Efficient Method to Study the Tradeoff Between Power Amplifier Efficiency and Digital Predistortion Complexity. IEEE Microwave and Wireless Components Letters, 2019, 29 (11), pp.741 - 744. 10.1109/LMWC.2019.2939911 . hal-02054967v2

HAL Id: hal-02054967

https://hal-centralesupelec.archives-ouvertes.fr/hal-02054967v2

Submitted on 6 Dec 2019

HAL is a multi-disciplinary open access archive for the deposit and dissemination of scientific research documents, whether they are published or not. The documents may come from teaching and research institutions in France or abroad, or from public or private research centers.
L'archive ouverte pluridisciplinaire HAL, est destinée au dépôt et à la diffusion de documents scientifiques de niveau recherche, publiés ou non, émanant des établissements d'enseignement et de recherche français ou étrangers, des laboratoires publics ou privés. 


\title{
An Efficient Method to Study the Trade-off between Power Amplifier Efficiency and Digital Predistortion Complexity
}

\author{
Siqi Wang, Morgan Roger, Julien Sarrazin, Member, IEEE, and Caroline Lelandais-Perrault
}

\begin{abstract}
This paper proposes a method to study the trade-off between the power amplifier (PA) power efficiency and the corresponding digital predistortion (DPD) model complexity needed for its linearization. The linearization performance estimated with the adjacent channel power ratio (ACPR) is treated as a control variable. In order to study at different $\mathrm{PA}$ operating points (OP), an algorithm is proposed to trace approximate Pareto fronts of the ACPR versus the DPD model number of coefficients. Crest factor reduction is applied when the backoff of the OP is less than the peak-to-average power ratio (PAPR) of the Long Term Evolution (LTE) signal. Experimental results on a PA with an LTE signal give interesting insights on the relation between DPD complexity and PA power efficiency.
\end{abstract}

Index Terms-Crest factor reduction, digital predistortion, nonlinear distortion, power amplifiers, power efficiency

\section{INTRODUCTION}

D IGITAL predistortion (DPD) is a common method to compensate for nonlinearities of radio frequency (RF) power amplifiers (PA) allowing it to work in high power efficiency zone near saturation [1]. This is typically advantageous when the PA output power is high enough so that the DPD power consumption is negligible.

However, in modern telecommunication systems, the PA output power requirements may vary. In cases where the DPD power consumption might become non-negligible, one can look for the best trade-off from the PA operating point (OP) of view. Since the DPD is applied to save power by increasing the PA efficiency, the power consumed by the DPD needs to be assessed with respect to the saved power [2]. Also, at the first order, the DPD power consumption is related to the number of its model coefficients [3].

DPD and crest factor reduction (CFR) techniques control the PA power efficiency [4] by allowing to adjust the PA OP. Different OPs lead to different PA characteristics and thus the DPD model structure should be correspondingly adapted so that the linearization performance remains the same.

Manuscript received July 3, 2019; accepted August 30, 2019. (Corresponding author: $S$. Wang.)

S. Wang, M. Roger, C. Lelandais-Perrault are with GeePs - Group of electrical engineering - Paris, CNRS, CentraleSupélec, Univ. Paris-Sud, Université Paris-Saclay, Sorbonne Université, 3 \& 11 rue Joliot-Curie, Plateau de Moulon 91192 Gif-sur-Yvette CEDEX, France (e-mail of authors: firstname.surname@centralesupelec.fr).

J. Sarrazin is with the Sorbonne Université, Laboratory of Electronics and Electromagnetism, L2E, F-75005 Paris, France (e-mail: julien.sarrazin@upmc.fr).

Color versions of one or more of the figures in this paper are available online at http://ieeexplore.ieee.org.
In this paper, the final goal is to make a study on the trade-off between PA power efficiency and the necessary DPD complexity to achieve a given adjacent channel power ratio (ACPR) requirement. To do that, we propose an algorithm to estimate the minimum number of DPD coefficients at a chosen PA OP for different ACPR requirements. Then we apply this algorithm on data acquired from a PA at different OPs and visualize the relation between PA power efficiency and DPD complexity.

This paper is organized as follows. Section II presents the DPD model. An algorithm is introduced to plot approximate Pareto fronts for the DPD complexity versus its linearization performance in Section III. In section IV, the PA efficiency is measured and the DPD complexity is estimated according to the experimental results. Finally, section $\mathrm{V}$ gives a conclusion.

\section{Crest Factor Reduction and Digital PREDISTORTION}

The system architecture is illustrated in Fig 1, where, the output signals of the CFR and the DPD are denoted by $u_{c}(n)$ and $x(n)$ respectively. In cases where the OP has an input back-off less than the input signal peak-to-average power ratio (PAPR), the clip-and-filter method [5] is used in this paper as CFR technique with the number of iterations set at 10 as suggested in [4].

Regarding DPD identification, we use the indirect learning architecture to estimate PA post-inverse model coefficients as in Fig 1. Numerous DPD models have been proposed based on Volterra Series, e.g. generalized memory polynomial (GMP) [1], dynamic-deviation-reduction (DDR) model [6] and decomposed vector rotation-based behavioral model (DVR) [7], etc. We use the GMP in this paper since it exhibits good trade-off between modeling accuracy and model complexity [3]. The post-inverse output $z_{p}(n)$ modeled with the GMP can be written as:

$$
\begin{aligned}
z_{p}(n)= & \sum_{k=0}^{\mathcal{K}_{a}-1} \sum_{l=0}^{\mathcal{L}_{a}-1} a_{k l} z(n-l)|z(n-l)|^{k} \\
& +\sum_{k=1}^{\mathcal{K}_{b}} \sum_{l=0}^{\mathcal{L}_{b}-1} \sum_{m=1}^{\mathcal{M}_{b}} b_{k l m} z(n-l)|z(n-l-m)|^{k} \\
& +\sum_{k=1}^{\mathcal{K}_{c}} \sum_{l=0}^{\mathcal{L}_{c}-1} \sum_{m=1}^{\mathcal{M}_{c}} c_{k l m} z(n-l)|z(n-l+m)|^{k}
\end{aligned}
$$

where the input signal is $z(n)$ which is the PA output $y(n)$ divided by $g$, the nominal gain of PA, $k$ is the index for 


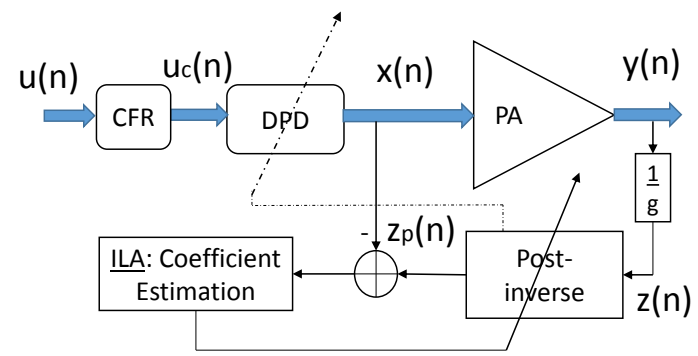

Fig. 1. Indirect learning architecture (ILA)
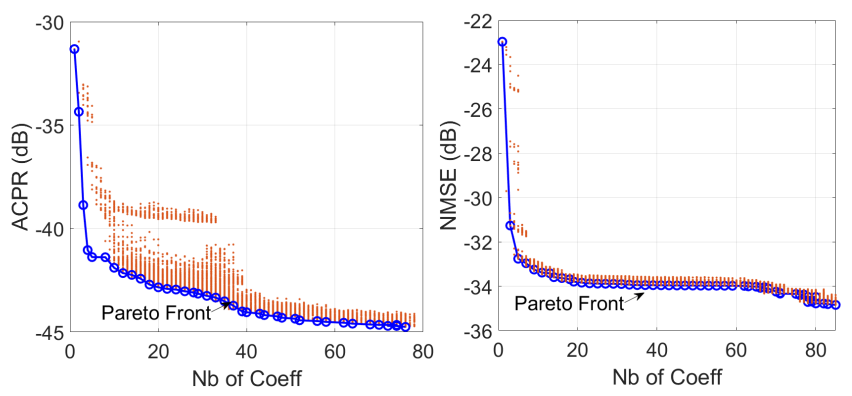

Fig. 2. The proposed algorithm traces out an approximate Pareto Front composed of optimal models

nonlinearity, and $l, m$ are the indices for memory, $\mathcal{K}_{a}, \mathcal{K}_{b}$, $\mathcal{K}_{c}$ are the highest orders of nonlinearity. $\mathcal{L}_{a}, \mathcal{L}_{b}, \mathcal{L}_{c}$ are the highest memory depths. $\mathcal{M}_{b}, \mathcal{M}_{c}$ denote the longest lagging and leading delay tap length, respectively. $a_{k l}, b_{k l m}, c_{k l m}$ are the complex coefficients of the signal and envelope, the signal and lagging envelope, and the signal and leading envelope, respectively. These coefficients can be estimated using least squares (LS) as in [8] by solving:

$$
\left[\mathbf{Z}^{H} \mathbf{Z}\right] \hat{\boldsymbol{c}}=\mathbf{Z}^{H} \boldsymbol{x}
$$

which minimizes the cost function

$$
C=\sum_{n=1}^{N}\left|z_{p}(n)-x(n)\right|^{2} .
$$

where $\boldsymbol{x}$ is the PA input, $\boldsymbol{Z}$ is the $N \times R$ matrix containing basis functions of $\boldsymbol{z}, \boldsymbol{c}$ is a $R \times 1$ vector containing $a_{k l}, b_{k l m}$, $c_{k l m}, R$ is the total number of coefficients.

\section{Algorithm FOR OPtimal SOLUTIONS}

According to (1), we can see that, by varying values of $\left(\mathcal{K}_{a}, \mathcal{L}_{a}, \ldots, \mathcal{M}_{c}\right)$, DPD models with the same number of coefficients may correspond to different structures thus different linearization performance.

The linearization performance is evaluated here with the ACPR of the post-inverse output $z_{p}(n)$. It could also be evaluated with the normalized mean square error (NMSE) or error vector magnitude (EVM) between $z_{p}(n)$ and $x(n)$. In order to know the necessary DPD complexity, we need to find out for each number of coefficients the corresponding DPD model with the best ACPR values.

In [8], an algorithm based on the Hill-Climbing heuristic has been proposed to determine an optimal DPD structure. It mainly addresses solving an optimization problem within a short time.

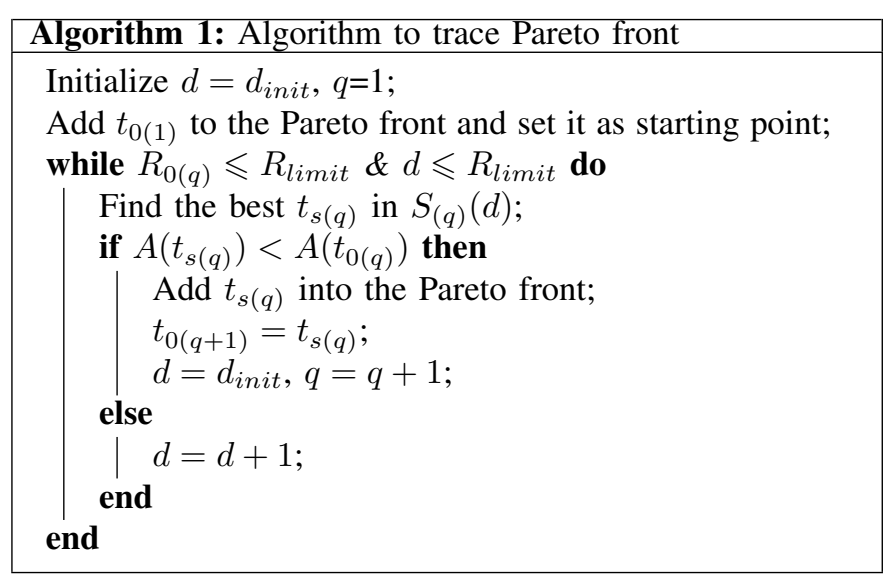

Algorithm 1 summarizes the modified version proposed in this paper and detailed below. It allows users to obtain all optimal solutions with different number of coefficients and to choose the most appropriate model structure according to their customized criterion. It gives out all models with the lowest ACPR/NMSE values as the blue curve in Fig 2 without doing an exhaustive search. The red points are the models tested in the algorithm. The blue circles are Pareto-optimal points such that there is no point who outperforms them on both ACPR and number of coefficients. The set of Pareto-optimal solutions is called Pareto front [9].

We define a neighbor $t_{j}$ of the model $t_{i}$ as

1) an 8-tuple $\left(\mathcal{K}_{a, i}+\delta_{1}, \mathcal{L}_{a, i}+\delta_{2}, \ldots, \mathcal{M}_{c, i}+\delta_{8}\right)$, where $\delta_{1, \ldots, 8} \in[0, \pm 1]$ and $\delta_{1, \ldots, 8}$ are not 0 at the same time.

2) $\left|R_{i}-R_{j}\right|<d$ where $d$ is a constraint on the number of coefficients.

The subspace of neighbors is denoted by $S(d)$.

The first starting point $t_{0}$ is the simplest model. The constraint $d$ is initialized at $d_{\text {init }}=2$ and it increases when there is no better element in $S(d)$. If a better neighbor $t_{s}$ is then found, $d$ is reset to its initial value. We set a constraint $R_{\text {limit }}$ on the total number of coefficients. 6658 and 6563 models were tested to plot the Pareto front for respectively ACPR and NMSE in Fig. 2. Using the exhaustive search, if each parameter is limited to 10 , there will be more than $10^{8}$ models to test. The study on the relation between PA efficiency and its DPD complexity needs to explore a large amount of OPs. Using the proposed algorithm can save enormous time.

\section{EXPERIMENTAL RESULTS}

With the algorithm proposed in Section III, we can quickly obtain the DPD models with best ACPR or NMSE values at different PA OPs. The PA under test is a HMC409LP4E PA fabricated by Analog Devices. Its nominal gain at $3.5 \mathrm{GHz}$ is $31 \mathrm{~dB}$ and the saturated output power is $32.5 \mathrm{dBm}$. The supply voltage is $5 \mathrm{~V}$. We generate the modulated signal with a carrier frequency of $3.5 \mathrm{GHz}$ in the PC Workstation and feed it to the PA through an Arbitrary Waveform Generator (AWG) with $10 \mathrm{GHz}$ sampling frequency. The input and output baseband signals are synchronized after down-sampling to $120 \mathrm{MHz}$ to be used by the identification algorithm. 


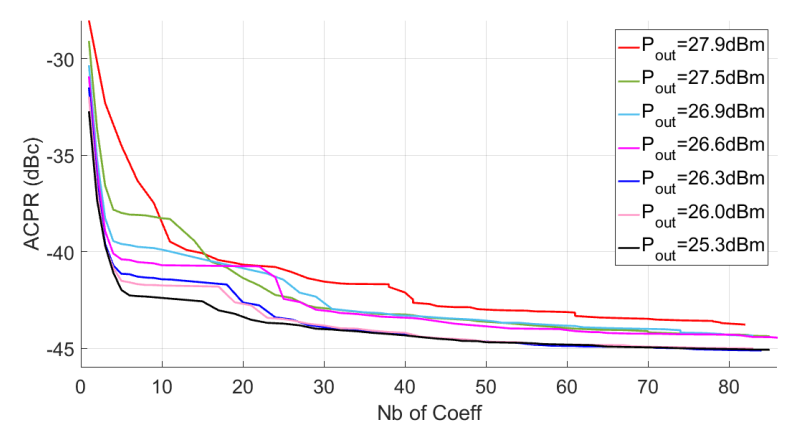

Fig. 3. Pareto fronts of the ACPR versus the number of DPD coefficients for different PA OPs

TABLE I

IMPACT OF THE PA OP ON PA EFFICIENCY AND NECESSARY NUMBER OF DPD COEFFICIENTS FOR DIFFERENT ACPR REQUIREMENTS

\begin{tabular}{|c|c|c|c|c|c|c|c|c|}
\hline \multicolumn{2}{|c|}{$P_{i n}(\mathrm{dBm})$} & -1.0 & -0.1 & 0.6 & 1.1 & 1.2 & 2.6 & 3.6 \\
\hline \multicolumn{2}{|c|}{$I_{\text {supply }}(\mathrm{A})$} & 0.73 & 0.77 & 0.79 & 0.81 & 0.83 & 0.87 & 0.92 \\
\hline \multicolumn{2}{|c|}{$P_{\text {out }}(\mathrm{dBm})$} & 25.3 & 26.0 & 26.3 & 26.6 & 26.9 & 27.5 & 27.9 \\
\hline \multicolumn{2}{|c|}{ PAE (\%) } & 9.3 & 10.4 & 10.8 & 11.4 & 11.9 & 12.8 & 13.4 \\
\hline \multirow{9}{*}{$\mathrm{R}$} & A:-37 & 1.9 & 2.1 & 2.2 & 2.4 & 2.6 & 3.4 & 8.2 \\
\hline & A:-39 & 2.7 & 2.8 & 2.9 & 3.1 & 3.7 & 12.8 & 10.5 \\
\hline & A:-41 & 3.8 & 4.2 & 4.7 & 23.1 & 21.6 & 18.9 & 25.8 \\
\hline & A:-42 & 5.2 & 17.5 & 18.8 & 25.0 & 27.0 & 23.0 & 39.2 \\
\hline & A:-43 & 16.8 & 21.8 & 22.9 & 29.2 & 31.8 & 31.8 & 49.5 \\
\hline & A:-43.5 & 20.9 & 25.6 & 26.5 & 42.4 & 47.8 & 46.5 & 70.7 \\
\hline & A:-43.7 & 23.8 & 27.4 & 27.8 & 45.7 & 54.7 & 52.6 & 79.1 \\
\hline & A:-44 & 29.6 & 31.3 & 34.5 & 57.5 & 70.3 & 61.7 & - \\
\hline & A:-44.3 & 38.9 & 40.4 & 41.2 & 80.3 & 79.7 & 82.0 & - \\
\hline
\end{tabular}

A: ACPR requirements in $\mathrm{dBc}$.

R: Number of DPD coefficients.

TABLE II

IMPACT OF THE PA OP ON PA EFFICIENCY AND NECESSARY NUMBER OF DPD COEFFICIENTS FOR DIFFERENT NMSE REQUIREMENTS

\begin{tabular}{|c|c|c|c|c|c|c|c|c|}
\hline \multicolumn{2}{|c|}{ PAE (\%) } & 9.3 & 10.4 & 10.8 & 11.4 & 11.9 & 12.8 & 13.4 \\
\hline \multirow{4}{*}{$\mathrm{R}$} & NE:-30 & 2.7 & 2.7 & 2.7 & 2.8 & 2.9 & 5.3 & 20.7 \\
\cline { 2 - 9 } & NE:-32 & 8.3 & 5.8 & 4.5 & 4.0 & 5.9 & 10.9 & - \\
\cline { 2 - 9 } & NE:-33 & 15.3 & 17.3 & 15.3 & 7.2 & 62.4 & 21.2 & - \\
\cline { 2 - 9 } & NE:-34 & 24.2 & 31.6 & 40.5 & 62.5 & - & 82.0 & - \\
\hline
\end{tabular}

NE: NMSE level in $\mathrm{dB}$.

R: Number of DPD coefficients.

A $20 \mathrm{MHz}$ LTE signal with 614400 samples is used as stimulus and 25000 samples are used in iterative DPD identifications. Its PAPR at $10^{-4}$ probability level is $8 \mathrm{~dB}$.

We use as a measure of efficiency the PA power-added efficiency (PAE). For different PA OPs, e.g. the average output power varying between $25.3 \mathrm{dBm}$ and $27.9 \mathrm{dBm}$, we apply the algorithm proposed in Section III to obtain the Pareto fronts as illustrated in Fig 3 and Fig 4 for ACPR and NMSE respectively. The CFR technique described in Section II is applied to keep signal samples out of the PA saturation zone.

In order to study the relation between the PAE and the DPD complexity, we treat the ACPR or NMSE value as a control variable. Thus we make a linear interpolation to estimate the number of coefficients corresponding to a given ACPR or NMSE and we keep decimals to preserve the accuracy. Table I gives the PA efficiency and the necessary number of DPD coefficients for different ACPR requirements at different PA OPs.

The DPD complexity versus the PA efficiency is then plotted

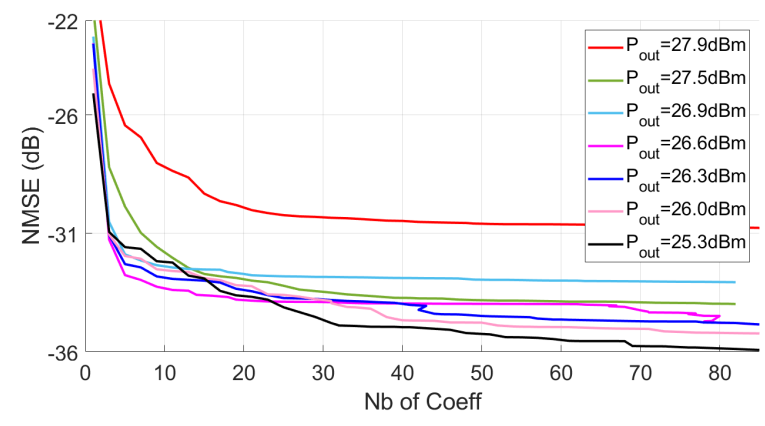

Fig. 4. Pareto fronts of the NMSE versus the number of DPD coefficients for different PA OPs

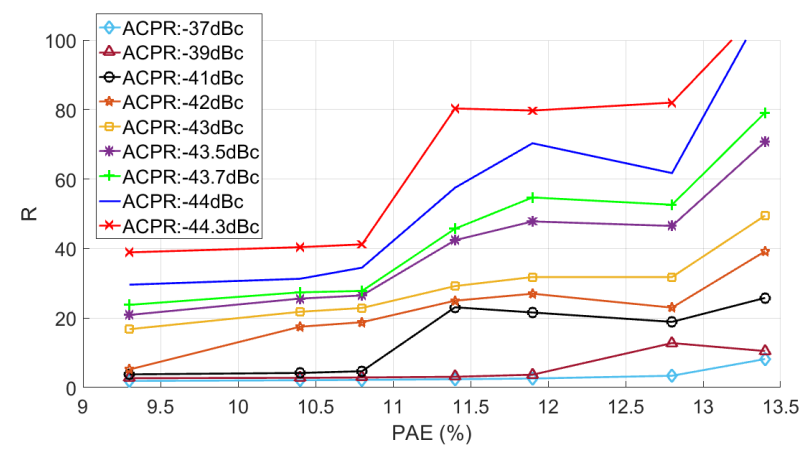

Fig. 5. DPD complexity vs PA efficiency

in Fig 5. For the tested PA, the higher the ACPR requirement is, the larger dynamic of the DPD complexity is versus the PA efficiency. This means that the DPD complexity limits more on the PAE when the ACPR requirement becomes strict.

Thanks to this method, the system designers will be able to optimize their choices. For instance, with a high ACPR requirement, it may not be worth to increase the PAE of the tested PA up to $13.4 \%$ considering the added DPD complexity.

For loose ACPR requirements, the PAE does not influence much the necessary DPD complexity. On the other hand, for strict ACPR requirements, the DPD complexity and consequently the DPD power consumption increases rapidly with the PAE, meaning the optimal OP w.r.t. the overall power consumption needs to be further studied.

The results for the NMSE as the control variable are given in Table II. We can see that the NMSE is strictly kept lower than $-30 \mathrm{~dB}$ when the DPD has more than 20 coefficients.

\section{CONCLUSion}

In this paper, an modified algorithm is proposed to trace approximate Pareto fronts for studying the trade-off between DPD model complexity and its linearization performance. It then helps to study the influence of the PA OP on the PA power efficiency and the necessary number of DPD coefficients for different ACPR or NMSE requirements with a reduced number of tests compared to the exhaustive search. Measurements with a $20 \mathrm{MHz}$ LTE stimulus show that the trade-off between the power saved from the PA dissipation and the DPD power consumption heavily depends on the ACPR requirement: the more stringent it is, the more room there is to optimize the trade-off. 


\section{REFERENCES}

[1] D. Morgan, Z. Ma, J. Kim, M. Zierdt, and J. Pastalan, "A generalized memory polynomial model for digital predistortion of rf power amplifiers," IEEE Trans. Signal Process., vol. 54, no. 10, pp. 3852-3860, Oct. 2006.

[2] S. Boumard, M. Lasanen, O. Apilo, A. Hekkala, C. Cassan, J. P. Verdeil, J. David, and L. Pichon, "Power consumption trade-off between power amplifier obo, dpd, and clipping and filtering," in 2014 26th International Teletraffic Congress (ITC), Sep. 2014, pp. 1-5.

[3] A. S. Tehrani, H. Cao, S. Afsardoost, T. Eriksson, M. Isaksson, and C. Fager, "A comparative analysis of the complexity/accuracy tradeoff in power amplifier behavioral models," IEEE Trans. Microw. Theory Techn., vol. 58, no. 6, pp. 1510-1520, Jun. 2010.

[4] S. Wang, M. Roger, and C. Lelandais-Perrault, "Impacts of crest factor reduction and digital predistortion on linearity and power efficiency of power amplifiers," IEEE Trans. Circuits Syst. II, Exp. Briefs, vol. 66, no. 3, pp. 407-411, Mar. 2019.

[5] H. Enzinger, K. Freiberger, and C. Vogel, "Competitive linearity for envelope tracking: Dual-band crest factor reduction and $2 \mathrm{~d}$-vector-switched digital predistortion," IEEE Microw. Mag., vol. 19, no. 1, pp. 69-77, Jan. 2018.

[6] A. Zhu, J. Pedro, and T. Brazil, "Dynamic deviation reduction-based volterra behavioral modeling of rf power amplifiers," IEEE Trans. Microw. Theory Techn., vol. 54, no. 12, pp. 4323-4332, Dec. 2006.

[7] A. Zhu, "Decomposed vector rotation-based behavioral modeling for digital predistortion of rf power amplifiers," IEEE Trans. Microw. Theory Techn., vol. 63, no. 2, pp. 737-744, Feb. 2015.

[8] S. Wang, M. A. Hussein, O. Venard, and G. Baudoin, "A novel algorithm for determining the structure of digital predistortion models," IEEE Trans. Veh. Technol., vol. 67, no. 8, pp. 7326-7340, Aug. 2018.

[9] X. B. Hu, M. Wang, and E. D. Paolo, "Calculating complete and exact pareto front for multiobjective optimization: A new deterministic approach for discrete problems," IEEE Trans. Cybern., vol. 43, no. 3, pp. 1088-1101, Jun. 2013. 